\title{
Experiencia docente universitaria mediante el uso de metodologías activas para el aprendizaje como la evaluación formativa compartida y el trabajo de competencias transversales.
}

\author{
Sara Trigueros Preciado ${ }^{a}$, Daniel Pérez González ${ }^{\mathrm{b}}$, Pedro Solana González ${ }^{\mathrm{c}}$ y Elena García \\ Ruiz $^{\text {d }}$ \\ ${ }^{a}$ Profesora en Facultad de CC. Económicas y Empresariales (Universidad de Cantabria, \\ sara.trigueros@unican.es), brofesor en Facultad de CC. Económicas y Empresariales (Universidad de \\ Cantabria, daniel.perez@unican.es), ${ }^{c}$ Profesor en Facultad de CC. Económicas y Empresariales \\ (Universidad de Cantabria, pedro.solana@unican.es) y ${ }^{\mathrm{d}}$ Profesora en Facultad de CC. Económicas y \\ Empresariales (Universidad de Cantabria, elena.garcia@unican.es).
}

\begin{abstract}
This work shows an active methodology for university teaching through cooperative learning and learning assessment conducted through the collaborative formative assessment, all mediated by the transversal skills of teamwork and communicative of public speaking.

The research methodology consisted of comparing the marks obtained in the same subject prior to implement the cooperative learning and collaborative formative assessment with the ones obtained after their use. The results show that the use of tools of cooperative learning and participatory and formative assessment originate better learning in students in relation to older and more traditional teaching-learning and assessment methods, also increases their involvement and learning, taken advantage of those to further enhance their skills of teamwork and public speaking communication.
\end{abstract}

Keywords: Cooperative learning, participative and formative evaluation, university teaching, transversal skills, public speaking, teamwork, shared formative assessment, European Higher Education Area, rubric.

\footnotetext{
Resumen

Este trabajo muestra una metodología activa para la enseñanza universitaria a través del aprendizaje cooperativo y la evaluación de los aprendizajes realizada a través de la evaluación formativa compartida, todo ello vehiculizado con las competencias transversales de trabajo en equipo y comunicativa de hablar en público.

La metodología de investigación ha consistido en comparar las calificaciones obtenidas en la misma asignatura con anterioridad a implementar el aprendizaje cooperativo y la evaluación compartida
} 
formativa, con las obtenidas después de utilizarla. Los resultados demuestran que la utilización de herramientas de aprendizaje cooperativo y de evaluación participativa y formativa originan un mejor aprendizaje en el alumnado en relación a metodologías más antiguas y tradicionales de enseñanza-aprendizaje y evaluación, esta nueva metodología aumenta su implicación y aprendizaje, la cual se aprovecha para potenciar asimismo sus competencias de trabajo en equipo y comunicativa de hablar en público.

Palabras clave: Aprendizaje cooperativo, evaluación participativa y formativa, docencia universitaria, competencias transversales, hablar en público, trabajo en equipo, evaluación formativa compartida, Espacio Europeo de Educación Superior, rúbrica.

\section{Introducción}

La convergencia Europea en Educación Superior está en conseguir estructuras más flexibles y un nuevo enfoque basado en el aprendizaje. Este nuevo modelo de universidad se centra en una concepción del aprendizaje constructivista, en el que el estudiante es el eje central en la creación de significado y el docente mediador entre el conocimiento y el alumnado (Olmedo, 2013), por eso en este trabajo llevado a cabo en una asignatura de postgrado, planteamos una metodología de clase en la que se conjugan y ponen de manifiesto varias metodologías activas para el aprendizaje en la universidad, como son el aprendizaje cooperativo a través del trabajo en equipo, la evaluación formativa compartida, además utilizando, y por tanto reforzando, las competencias transversales de trabajo en equipo y de comunicación para hablar en público. Es decir, implementamos la evaluación en la que participan los estudiantes recíprocamente y la evaluación formativa compartida con el profesor para conseguir un mejor aprendizaje y resultado, pero además aprovechándonos de las bondades de este tipo de aprendizaje y evaluación, como es el aumento de implicación de los estudiantes (Gessa 2011; Ureña y Ruiz, 2012; Hortigüela-Alcalá, Pérez-Pueyo y López-Pastor, 2015) lo utilizamos para además conseguir que sirva para formarles, siendo también como un tipo de evaluación formativa compartida donde los resultados se comparten con el profesor, y desarrollaran competencias transversales como son el trabajo en equipo y la comunicación para hablar en público, colaborando de esta manera con el compromiso de desarrollar una experiencia de evaluación formativa y compartida orientándola al desarrollo de competencias profesionales en la línea del modelo ECTS (European Credit Transfer System) y plasmando en este documento los resultados obtenidos (López, Martínez y Julián, 2007; Hamodi, López, y López, 2014).

Se fomenta así la creación de contextos de aprendizaje basados en la comunicación, potenciando la creación de grupos de elaboración del conocimiento que tienen como objeto ayudar a los alumnos a "buscar el aprendizaje como finalidad de forma activa y estratégica" (Scardamalia y Bereiter, 


\section{Objetivos}

Los objetivos que aquí nos planteamos son, que a través de la metodología activa y participativa de clase en este trabajo expuesta, los estudiantes adquieran un mayor y mejor aprendizaje, que se sientan parte integrante y activa del desarrollo de las lecciones, obteniendo mejores resultados calificatorios (Alvarado y García Jiménez, 1997; García Alvarado y Jiménez, 2000).

Todo ello se medirá y cuantificará con las calificaciones finales obtenidas en la asignatura, las cuales señalarán, si son altas, que el aprendizaje y rendimiento académico ha sido superior (Edel, 2003, López et al, 2012). Además se compararán con las obtenidas en años previos, cuando estas técnicas aún no habían sido implementadas, para demostrar esas mejoras buscadas (Fraile et al, 2013).

\section{Desarrollo de la Innovación}

Numerosas tendencias e investigaciones en educación nos señalan la necesidad a cambiar las formas de enseñanza y evaluación utilizadas tradicionalmente en el espacio de enseñanza, y proponen otras metodologías más exitosas para conseguir mejores resultados y adaptarnos al nuevo espacio propuesto por Bolonia (Arribas, Carabias y Monreal, 2010; Gessa 2011; Ureña y Ruiz, 2012; Fraile et al, 2013; Rodríguez et al, 2013; Hamodi, López, y López, 2014; Nuñez et al, 2014; Hamodi, López, y López, 2015; Hortigüela-Alcalá, Pérez-Pueyo y López-Pastor, 2015).

Por eso nos proponemos una metodología integradora que conjugue varias formas activas de aprendizaje y evaluación que pasaremos a explicar a continuación.

Para comenzar, diremos que en el diseño y elaboración de la clase utilizaremos el aprendizaje cooperativo y el trabajo en equipo. En el aprendizaje cooperativo los estudiantes que trabajan aprenden juntos, se implican más activamente en el proceso de aprendizaje (Cuseo, 1996) puesto que permite a los estudiantes actuar sobre su propio proceso de aprendizaje, implicándose más con la materia de estudio y con sus compañeros (Domingo, 2008). Elegimos este tipo de aprendizaje, porque a través de los métodos y técnicas de aprendizaje cooperativo, se logran cinco elementos esenciales: interdependencia positiva, interacción cara a cara, responsabilidad individual, habilidades sociales y el procesamiento grupal autónomo (Johnson y Johnson, 1987).

Concretamente en nuestro trabajo utilizaremos la técnica Grupo de investigación de (Sharan, 1980) donde los estudiantes escogen subtemas de la materia que tienen que trabajar en clase. Cada grupo divide los subtemas en tareas individuales dentro del grupo, para posteriormente poner en común y finalizar las tareas juntos y después presentan los resultados a toda la clase. Particularmente, en nuestro estudio, posteriormente a que el profesor les explique la materia se les propone realizar durante la clase un trabajo práctico en grupo, en el que diseñan un ejemplo, incluyendo una serie de apartados que cubren los contenidos vistos, y que ellos dentro de su grupo se reparten.

(cc) BY-NC-ND 2016, Universitat Politècnica de València 
Después tendrán que hacer un power point y lo expondrán delante de la totalidad de la clase, participando todo el grupo junto, repartiéndose los roles de un equipo. De este modo estamos utilizando la metodología de aprendizaje cooperativo, trabajando la competencia de trabajo en equipo y de hablar en público.

Hemos querido potenciar estas competencias transversales porque preparan a los estudiantes para el mundo de trabajo actual, porque los recién titulados presentan carencias en su capacidad para trabajar en grupo (Domingo, 2008; Chica, 2011), siguiendo asimismo la recomendación del Plan Bolonia del Espacio Europeo de Educación Superior de centralizar el proceso de enseñanza aprendizaje, dotando al alumnado de competencias que le faculten a la hora de finalizar sus estudios para el desempeño profesional (MEC, 2003). Facilitando también una pedagogía consistente en el contexto laboral que encontrarán los estudiantes al titularse, trabajando habilidades interpersonales (como la negociación, el consenso, el respeto, la capacidad para comprender los puntos de vista de los demás, argumentar estructuradamente y de forma lógica y coherente los propios, expresarse con corrección, criticar sin herir, etc.) que se obtienen si se tiene la ocasión de practicarlos (Domingo, 2008; Chica, 2011; Morales y Navarro, 2013) y que mejor practicarlo en un máster que prepara y especializa para formar expertos de alto nivel con la finalidad de que adquieran una formación avanzada, orientada a la especialización profesional (Ureña y Ruiz, 2012).

Hemos explicado y justificado ya que el aprendizaje será cooperativo y porqué, pero además hemos dicho que será trabajo en equipo, ahora detallaremos que para que se del trabajo en equipo dentro de un grupo de personas según Domingo (2008), se deben dar las siguientes condiciones:

a) Ser un grupo estructurado (con roles) con unos objetivos claros, conocidos y compartidos por todos, con los que se identifican todos los componentes del grupo.

Los objetivos del equipo están claros, consisten en realizar el trabajo, más arriba explicado, propuesto por el profesor que contará para nota y que será calificado a todo el grupo en su conjunto y no individualmente a cada estudiante, luego el equipo tiene que trabajar coordinadamente para conseguir su mismo objetivo final. Cada integrante se encargará de una tarea del trabajo con lo que los roles vienen intrínsecamente dados, ya que después de haber elegido y consensuado el tema y proyecto que realizarán, un miembro por ejemplo, se encargará de la introducción, otro del desarrollo, otro de conclusiones, otro de buscar bibliografía, imágenes, otro de hacer la ppt, etc.

b) Que exista interdependencia positiva, es decir que se necesiten y que reconozcan esta necesidad mutua de cara a lograr el objetivo común.

La interdependencia positiva también está garantizada debido a que deben trabajar todos los integrantes la parte que se hayan repartido para que dé tiempo a finalizarlo en el tiempo disponible que son dos sesiones de 4 horas cada una. En la última sesión se destinará 1 hora, la última, para la exposición y evaluación de los trabajos por los equipos. 
c) Que exista un coordinador que asuma el liderazgo del grupo (puede ser electo, por rotación, por designación u otros).

El rol de coordinador será rotativo en función de la descarga y consecución de tareas, es decir, los integrantes según vayan terminando su parte asignada, harán el seguimiento del resto de tareas y de los resultados, del plazo disponible y proveerán e informarán a todos los componentes de los productos resultantes del trabajo en grupo.

Para Cardona y Wilkinson (2006) el trabajo en equipo requiere el ejercicio de una serie de capacidades esenciales como proponer ideas y aceptar las de otros, gestionar bien el tiempo, escuchar, colaborar, adaptarse, etc. Todas estas capacidades son llevadas a cabo por el alumnado objeto de este estudio, dado que la actividad propuesta así lo requiere. Para ello es importante que el equipo evite técnicas individualistas. Por eso los equipos para garantizarse un buen trabajo, utilizarán técnicas como las siguientes: tormenta de ideas, puestas en común, estudio de documentos, estudio de casos, discusiones de ideas y decisiones por consenso. Los grupos tendrán a su disposición materiales suministrados por el profesor, equipos informáticos y conexión a Internet para la profundización y elaboración del trabajo.

En cuanto a la otra competencia transversal comunicativa de hablar en público, comentar que la competencia de hablar en público, está incluida dentro de las capacidades cognitivas como destreza lingüística o capacidades para la comunicación oral y escrita. Y también lleva incluida como segunda competencia la relacionada con la inteligencia emocional, asociada a la facultad interpersonal relacionada con la expresión de sentimientos y con la capacidad crítica y autocrítica (Morales y Navarro, 2013).

La facultad de hablar es innata en el ser humano y se manifiesta a corta edad, pero se observa que cuando una persona necesita ponerla en práctica en un contexto determinado, formal y persiguiendo unos objetivos específicos requiere de un aprendizaje (Avendaño y Miretti, 2007; Morales y Navarro, 2013). Se percibe en el alumnado la inseguridad que manifiesta cuando tiene que hablar delante de un grupo de personas (Orejudo et al, 2007; Morales y Navarro, 2013). Y es que el miedo a hablar en público es uno de los problemas más común entre la población universitaria (Stein, Walker y Forde, 1996; Méndez, Inglés e Hidalgo, 1999; Morales y Navarro, 2013) Adicionalmente, dominar la comunicación oral de manera efectiva, es imprescindible para conseguir un buen desarrollo de una profesión (Castejón, 2001; Fernández, 2002; Forgas, 2003; Morales y Navarro, 2013).

Por eso en la metodología aquí presentada, pedimos a nuestros estudiantes que practiquen esta competencia y por tanto adquieran un aprendizaje sobre ella, a través de la exposición oral de su trabajo por todos los integrantes del equipo y delante de la totalidad de la clase. La clase dividida en equipos calificará mediante una rúbrica facilitada por el docente, la calidad del trabajo expuesto y la competencia oral del equipo. El docente asimismo puntuará con la misma rúbrica a los grupos. $\mathrm{Y}$ es aquí donde entra en juego la evaluación formativa compartida.

La evaluación es una parte sustancial de la enseñanza, que determina cómo aprenden los estudiantes y su rendimiento (Rodríguez et al, 2013). Es un aspecto que deja huella en los 
estudiantes en la Educación Superior (Boud y Associates, 2010) y sigue siendo uno de los apartados que usualmente más preocupan a los estudiantes (Olmedo, 2013). Durante los últimos años, todas las universidades españolas han estado inmersas en un proceso de renovación que implica la consolidación del proceso de convergencia hacia el Espacio Europeo de Educación Superior (EEES). Dichos cambios afectan tanto al alumnado como al profesorado universitario. El profesorado universitario se ha encontrado con una serie de desafíos a los que enfrentarse; uno de ellos es modificar los criterios y las estrategias de evaluación (Gessa, 2011; Rodríguez, Ibarra y García, 2013; Hamodi, López, y López, 2015). Y es que la evaluación es uno de los elementos que debe evolucionar para ajustarse a esta nueva forma de entender y desarrollar la docencia universitaria (Hamodi, López, y López, 2015). Por tanto instaurar nuevas formas como la autoevaluación, la evaluación entre iguales o la coevaluación (Gessa, 2011) se hace necesario. Numerosas investigaciones rompen con una tradición en la que el profesor es el actor principal y único en la evaluación de los aprendizajes y demuestran y reclaman la importancia de la participación activa de los estudiantes en los procesos de evaluación (Dochy, Segers y Sluijsmans, 1999; Falchikov, 2005; Rodriguez, Ibarra y Garcia, 2013)

Pues como dice Hall (1995) "la participación de los estudiantes y profesores en el proceso de evaluación hace posible que los estudiantes se valoren a sí mismos, al tiempo que permite a los profesores mantener el control necesario sobre las evaluaciones finales". En este punto comentar, que numerosos estudios indican que hay una mezcla entre los términos que se usan para referirse a la evaluación formativa y compartida y a lo que podría entenderse como tal (Gullickson, 2007; Álvarez, 2009; Rodriguez, Ibarra y Garcia, 2013; Hamodi, López, y López, 2015). Por eso nosotros para nuestro trabajo hemos estimado que todos los términos que hacen referencia a una evaluación participativa, compartida o colaborativa son sinónimos, al igual que los autores Dochy, Segers y Sluijsmans (1999), Falchikov (2005) y Rodriguez, Ibarra y Garcia (2013) y nos referiremos a la metodología de evaluación utilizada en nuestro estudio como evaluación compartida o participativa, porque evalúan estudiantes y profesores (Rodriguez, Ibarra y Garcia, 2013; Hamodi, López, y López, 2015). Entonces entendemos la evaluación compartida como el proceso dialógico, individual o grupal, que mantiene el profesorado con el alumnado sobre la evaluación de los procesos de enseñanza-aprendizaje que se han dado (Pérez, Julián y López, 2009). En nuestro estudio el diálogo, fue grupal a través de los equipos formados.

En cuanto a "evaluación formativa" quiere decir que utilizamos la evaluación para el aprendizaje, que es una evaluación formadora (Pérez, Julián y López, 2009; López Manrique y Valles, 2011; Castejón et al, 2011). La Red de Evaluación Formativa y Compartida entiende la evaluación formativa como "todo proceso de constatación, valoración y toma de decisiones cuya finalidad es optimizar el proceso de enseñanza-aprendizaje que tiene lugar, desde una perspectiva humanizadora y no como mero fin calificador" (Pérez, Julián y López, 2009). Es decir es una evaluación para reconocer y responder al aprendizaje del estudiante con el fin de reforzarlo durante el propio proceso (Brown y Pickford, 2013). En nuestra investigación el estar pendiente de la exposición del resto de equipos para tener que calificarles por los estudiantes, provoca que aumente su aprendizaje pues tienen que asociar lo que ven y escuchan de sus compañeros con sus propios conocimientos adquiridos, lo cual refuerza su aprendizaje pues es una tarea adicional que 
implica atención; también responsabilidad por tener que cumplir con el compromiso de calificar a sus compañeros, lo que aumenta la implicación del alumnado porque no es un mero espectador y sus decisiones influyen en el resto; y a la vez suma nueva información y conocimiento al propio, lo cual enriquece nuevamente el aprendizaje.

Una vez explicado lo que es la evaluación formativa compartida, que es la técnica utilizada en nuestro trabajo, pues los estudiantes participan en el proceso evaluador teniendo responsabilidad como el profesor, ya que tienen que puntuar a sus compañeros y su parte será tenida en cuenta en la ponderación final, como seguidamente detallaremos, lo cual les sirve para aprender también, al repasar y estar pendientes de los aspectos que deben puntuar en la rúbrica utilizada para ello, pasaremos a detallar la rúbrica utilizada, pues la evaluación será desarrollada a través de una rúbrica.

A continuación, pasamos a explicar porqué hemos utilizado la técnica de rúbrica para la evaluación. La hemos seleccionado, porque su utilización permite que el alumnado pueda ser evaluado de forma objetiva, y al mismo tiempo permite al profesorado exponer previamente los criterios con los que va evaluar un trabajo (López, 2002), situando al alumnado ante los elementos clave de su aprendizaje y evaluación (Chica, 2011). Además el recurso de la rúbrica para la evaluación formativa es valorado positivamente por los estudiantes (Conde y Pozuelo, 2007) porque permite la orientación y evaluación en la práctica, pues describe los criterios a tener en cuenta para valorar un trabajo (Wamba et al, 2007). Los alumnos juzgan la experiencia de trabajo en equipo a partir de la utilización de una rúbrica, y con ella se favorecen la capacidad de escucha, el compromiso con el trabajo final, la responsabilidad individual, la acogida de otras ideas y propuestas distintas a las propias, etc. (Chica, 2011) aspectos importantes y necesarios para el desarrollo de la competencia de trabajo en equipo comentada en párrafos superiores.

Seguidamente mostraremos la rúbrica utilizada en la evaluación formativa compartida empleada tanto por el docente como por los estudiantes, los cuales divididos en equipos tienen que calificar al resto de equipos y deben consensuar la puntuación asignada por ellos entre todos los miembros que integran su equipo. La práctica aquí señalada es aplicada a un curso de postgrado. En una asignatura con una carga lectiva de 20 horas, de las cuales 8 horas se destinan a desarrollar la metodología aquí indicada y las 12 horas restantes son impartidas mediante el método de clase magistral, las cuales se dan antes de la metodología participativa. La clase tiene 25 alumnos, en ésta los grupos están formados por 5 miembros, lo que hace que haya un total de 5 equipos. Comentar también, que se dice al alumnado que para asignar la calificación el docente, tendrá en cuenta también la puntuación que hacen y que no está permitido poner a todos la misma puntuación, para evitar que pongan igual nota a todos los grupos y comprometerles así a atender y hacer una evaluación honesta.

Para el diseño y elaboración de la rúbrica utilizada, hemos partido de los ejemplos de rúbrica aportados por Gatica y Uribarren (2013), donde se da una descripción general con los descriptores de logro sobre calidad, comprensión o dominio global y cada nivel se define claramente para que los estudiantes sepan lo que significa, adaptados para nuestro caso concreto.

A través de la técnica de la rúbrica diseñada, será evaluada el trabajo que tienen que desarrollar

(c)) EY-NG-ND 2016, Universitat Politècnica de València

Congreso In-Red (2016) 
los alumnos por medio del trabajo en equipo y aprendizaje cooperativo, la rúbrica elaborada específicamente para ese fin, se muestra al final de esta comunicación.

\section{Resultados}

Una vez planteados los objetivos del trabajo y descrita la metodología seguida se presentan los resultados. En este punto lo que haremos será comparar las calificaciones obtenidas en esta asignatura por los alumnos del postgrado en el 2014, cuando todavía no se había implementado la técnica aquí descrita, con las obtenidas en el 2015 en la misma asignatura de postgrado ya con la técnica implementada para analizar las mejoras buscadas, las cuales observaremos por el incremento de calificación. Comentar que la evaluación y puntuación obtenida en el trabajo conforma el 33,33\% de la nota final de la asignatura, y el 66,66\% restante la calificación del examen final, en el cual se pregunta a su vez contenidos desarrollados a través de la elaboración del trabajo descrito, estos contenidos dentro del examen vienen a ser la mitad de las preguntas del examen, y la otra mitad está compuesta por preguntas sobre la primera parte de la asignatura donde se explican los contenidos por medio de la técnica de clase magistral. Lo cual quiere decir, que el 33,33\% del compendio del examen también deriva de la metodología de enseñanza-aprendizaje aquí expuesta, pues son preguntas sobre los conceptos trabajados en esa parte. Esto quiere decir que el 66,66\% de la calificación final depende de esta metodología.

Tabla 1. Configuración de la calificación final de la asignatura

\begin{tabular}{|c|c|c|c|}
\cline { 2 - 4 } \multicolumn{1}{c|}{} & Trabajo descrito & \multicolumn{2}{|c|}{ Examen Final Teórico } \\
\hline $\begin{array}{c}\text { Peso en la } \\
\text { calificación } \\
\text { final }\end{array}$ & $33,33 \%$ & $66,66 \%$ & $\begin{array}{l}33,33 \% \text { Contenidos explicados } \\
\text { en las } 12 \text { primeras horas (clase } \\
\text { magistral) }\end{array}$ \\
\cline { 2 - 3 } & & $\begin{array}{l}33,33 \% \text { Contenidos trabajados en } \\
\text { las } 8 \text { restantes mediante la } \\
\text { metodología activa descrita. }\end{array}$ \\
\hline
\end{tabular}

De esto se deduce que el 66,66 \% de la nota depende de la enseñanza-aprendizaje llevada a cabo mediante la práctica activa de metodología de clase aquí desarrollada, es decir a través del trabajo cooperativo desarrollado en equipo y la evaluación formativa compartida expuesta, se alcanzará el 66,66\% de resultados en la calificación, puesto que es lógico pensar que si el aprendizaje ha sido significativo en la fase de trabajo y evaluación formativa compartida, implicará que la parte del examen relacionada tenga mejores resultados.

A continuación mostramos las calificaciones obtenidas en la asignatura en el año 2014 y 2015, para analizar esas mejorar buscadas. 
Gráfico 1. Comparativa de calificaciones obtenidas en el 2014 y 2015

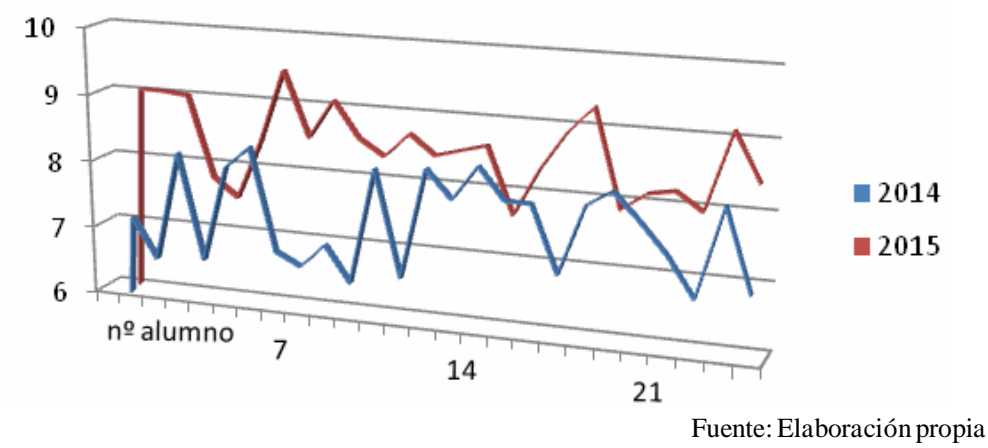

Gráfico 2. Comparativa del tipo de calificaciones obtenidas en el 2014 y 2015

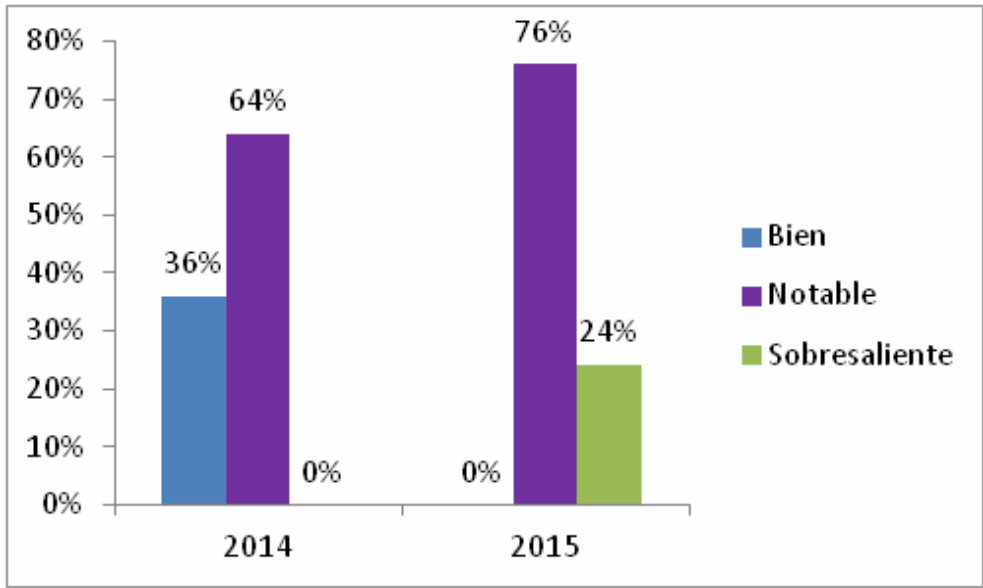

Fuente: Elaboración propia

Tabla 2. Comparativa de la Evolución de calificaciones entre el 2014 y 2015

\begin{tabular}{|c|c|c|c|}
\cline { 2 - 4 } \multicolumn{1}{c|}{} & $\mathbf{2 0 1 4}$ & $\mathbf{2 0 1 5}$ & $\Delta$ \\
\hline Bien & $36 \%$ & $0 \%$ & $-36 \% \downarrow$ \\
\hline Notable & $64 \%$ & $76 \%$ & $12 \% \uparrow$ \\
\hline Sobresaliente & $0 \%$ & $24 \%$ & $24 \% \uparrow$ \\
\hline Nota Máxima obtenida & 8,40 & 9,47 & $1,06 \uparrow$ \\
\hline Nota Mínima obtenida & 6,50 & 7,50 & $1,00 \uparrow$ \\
\hline Nota Media & 7,48 & 8,49 & $1,01 \uparrow$ \\
\hline
\end{tabular}

Fuente: Elaboración propia

Como podemos observar las calificaciones en el 2015 mejoran en comparación con las obtenidas en 2014. Se detecta un incremento del $12 \%$ en Notables, del $24 \%$ en Sobresalientes y una disminución del 36\% de Bienes, que han pasado a ser Notables y Sobresalientes en el

(c)) EY-NC-ND 2016, Universitat Politècnica de València 
2015. La nota media ha subido un punto respecto al 2014. Estos resultados indican una mejoría respecto al 2014, lo cual nos demuestran que la metodología propuesta mejora la calidad del proceso enseñanza-aprendizaje y su resultado.

\section{Conclusiones}

Los profesionales de la educación conocemos la importancia y complicación que tienen la aplicación de distintas metodologías en el aprendizaje de los alumnos, y en esta línea es necesario seguir desarrollando experiencias y compartirlas con la comunidad docente como vía para enriquecernos entre todos y seguir mejorando. En este sentido, este trabajo trata precisamente de exponer los resultados que se han alcanzado en una asignatura de postgrado oficial de la Universidad de Cantabria cuyo único cambio sustancial de un año al siguiente ha sido la metodología empleada. El análisis de los resultados derivados de aplicar una metodología del aprendizaje cooperativo, trabajo en equipo y la evaluación de los aprendizajes realizada a través de la evaluación formativa compartida han dado lugar a una mejor adquisición de competencias y un mayor aprendizaje que finalmente se ha plasmado en mejores resultados académicos. Puesto que es la misma asignatura, el mismo temario, mismo perfil de alumnos, y la única diferencia es la metodología activa de clase expuesta en esta comunicación, la cual difiere de la anterior metodología perteneciente al año previo, en introducir el trabajo en equipo y la evaluación formativa compartida, la cual a su vez también tiene en cuenta no solo los contenidos del trabajo, sino también las habilidades comunicativas de hablar en público (ver rúbrica al final), potenciando por tanto esta destreza, pues los estudiantes saben que es calificada y tenida en cuenta en la ponderación final de la rúbrica. Además al participar los estudiantes en la calificación aumenta su responsabilidad e implicación pues el alumnado no es un mero espectador si no que tiene el deber de evaluar a sus iguales de manera justa, lo cual aumenta su compromiso pues sus decisiones afectan al resto.

Desde luego las nuevas metodologías implementadas suponen un esfuerzo adicional para los docentes y un cambio en la forma de trabajar de los estudiantes, pero sin duda los resultados de la experiencia aquí expuesta hacen que merezca la pena. Por supuesto, debe señalarse que este trabajo tiene como limitación el ser una experiencia que de momento solo se ha podido poner en práctica un año, si bien los resultados obtenidos son similares a otras experiencias afines recogidas por la literatura. Como futura línea de investigación, indicar que se seguirá en años sucesivos con la implementación de esta metodología en ésta y otras asignaturas y con mismos y otros perfiles de alumnos, lo que permitirá profundizar el estudio de este tema. Aunque los autores del trabajo consideramos que obtener mejores y más altas calificaciones implica mayor satisfacción para el alumnado, otra futura línea de investigación podría ser analizar la satisfacción de los estudiantes sobre la utilización de este tipo de metodologías para valorar cómo es percibida por ellos.

\section{Referencias}

ALVARADO, J. M. y GARCIA, M. V. (1997) "Incidencia de la asistencia a clase, del trabajo efectivo y de factores individuales sobre el rendimiento académico.” En López, A.M., López J.L. y Moreno R. Actas del V Congreso de Metodología de las C.C. Humanas y Sociales. Sevilla: pp. 683-686.

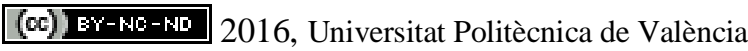

Congreso In-Red (2016) 
ÁlVAREZ, J. M. (2009). "La evaluación en la práctica de aula. Estudio de campo", Revista de Educación, núm. 350, pp. 351-374. < http://www.mecd.gob.es/revista-de-educacion/numeros-revistaeducacion/numeros-anteriores/2009/re350/re350_15.html> [Consulta: 22 de febrero 2016.]

ARRIBAS, J. M., CARABIAS, D. y MONREAL, I. (2010).” La docencia universitaria en la formación inicial del profesorado. El caso de la escuela de magisterio de Segovia." Revista Electrónica Interuniversitaria de Formación del Profesorado, 13 (3), 27-35.

<http://aufop.com/aufop/uploaded_files/articulos/1285861727.pdf> [Consulta: 10 de marzo 2016.]

AVENDAÑO, F. y MIRETTI, M. L. (2007). El desarrollo de la lengua oral en el aula. Estrategias para enseñar a escuchar y a hablar. Sevilla: Alcalá de Guadaíra.

BROWN, S. y PICKFORF, R. (2013), Evaluación de habilidades y competencias en educación superior. Madrid: Narcea.

CARDONA, P., y WILKINSON, H. (2006). “Trabajo en equipo”. Occasional Paper, 7(10), 3-4.

CASTEJÓN, F. J., LÓPEZ V., JULIÁN J. Y ZARAGOZA J. (2011), "Evaluación formativa y rendimiento académico en la formación inicial del profesorado de educación física", Revista Internacional de Medicina y Ciencias de la Actividad Física y el Deporte, vol. 11, núm. 42, 328-346,

< http://cdeporte.rediris.es/revista/revista42/artevaluacion163.htm> [Consulta en: 10 de diciembre de 2015]

CASTEJÓN, L. (2011). “¿Cómo desarrollar la competencia de hablar en público en el alumnado universitario?" Aula Abierta, 39 (3), 31-40.

<https://dialnet.unirioja.es/servlet/articulo?codigo=3691471 > [Consulta en: 10 de diciembre de 2015]

CHICA, E. (2011) "Una propuesta de evaluación para el trabajo en grupo mediante rúbrica." Escuela Abierta, 2011, 14, 67-81 < https://dialnet.unirioja.es/descarga/articulo/3896779.pdf> [Consulta: 12 de septiembre de 2014]

CONDE, A. y POZUELO, F. (2007). "Las plantillas de evaluación (rúbrica) como instrumento para la evaluación. Un estudio de caso en el marco de la reforma de la enseñanza universitaria en el EEES. " Investigación en la Escuela, 63, 77-90

CUSEO, J. B. (1996). “Cooperative learning: a pedagogy for addressing contemporary challenges \& critical issues in higher education”. New Forums Press.

DOCHY, F., SEGERS, M., SLUIJSMANS, D. (1999). "The Use of Self-, Peer and Co-assessment in Higher Education: a review." Studies in Higher Education, 24 (3), 331-350

DOMINGO, J (2008) "El aprendizaje cooperativo" Cuadernos de Trabajo Social, Vol. 21 (2008): 231-246

EDEL, R. (2003). "El rendimiento académico: concepto, investigación y desarrollo.” Revista Electrónica Iberoamericana sobre Calidad, Eficacia y Cambio en Educación, 1(2). 1-15

<http://www.ice.deusto.es/RINACE/reice/vol1n2/Edel.pdf> [Consulta: 10 septiembre 2015]

FRAILE, A. et al. (2013): "La evaluación formativa en docencia universitaria y el rendimiento académico del alumnado." Aula Abierta 41.2: 23-34.

GARCIA, M.V., ALVARADO, J. y JIMENEZ, A. (2000). "La predicción del rendimiento académico: regresión lineal versus regresión logística”. Psicothema, 12 (2), 248-252.

GATICA-LARA F. y URIBARREN-BERRUETA T. (2013) “¿Cómo elaborar una rúbrica?” Elsevier, Investigación en Educación Médica, 2(1), 61-65

〈http://riem.facmed.unam.mx/sites/all/archivos/V2Num01/10_PEM_GATICA.PDF〉 [Consulta: 12 de septiembre de 2014]

GESSA, A. (2011), "La coevaluación como metodología complementaria de la evaluación del aprendizaje.

(c) 2016, Universitat Politècnica de València

Congreso In-Red (2016) 
Análisis y reflexión en las aulas universitarias", Revista de Educación, núm. 354, 749- 764, < http://www.revistaeducacion.mec.es/re354/re354_30.pdf > [Consulta: 13 de octubre de 2015].

FALCHIKOV, N. (2005). "Improving Assessment Through Student Involvement." Practical solutions for aiding learning in higher and further education. London: Routledge-Falmer.

FERNANDEZ, A. M. (2002). "Habilidades para la comunicación y la competencia comunicativa." Comunicación Educativa. $2^{\mathrm{a}}$ ed. La Habana: Pueblo y Educación.

FORGAS, B. J. A. (2003). "Diseño curricular por competencias: Una alternativa para la formación de un técnico competente." En Colección Pedagogía. Instituto Superior Pedagógico Frank País. Palacio de las Convenciones de La Habana

GULLICKSON, A. (2007), Estándares para la evaluación de los aprendizajes de los estudiantes, Bilbao: Mensajero.

HAMODI, C., LOPEZ, A. T., LOPEZ, V. (2014). "Red de evaluación formativa y compartida en docencia universitaria: creación, consolidación y líneas de trabajo." Revista de evaluación educativa, 3,1 . < http://revalue.mx/revista/index.php/revalue/article/view/110 > [Consultado en: 11 de marzo de 2016]

HAMODI, C., LÓPEZ V., y LÓPEZ, A.T. (2015). "Medios, técnicas e instrumentos de evaluación formativa y compartida del aprendizaje en educación superior." Perfiles educativos 37.147 (2015): 146-161.

HORTIGÜELA-ALCALA, D., PEREZ-PUEYO, Á. y LOPEZ-PASTOR, V. (2015). "Implicación y regulación del trabajo del alumnado en los sistemas de evaluación formativa en educación superior." RELIEVE, 21, 1

IBARRA, M. S., RODRIGUEZ G. y GOMEZ M.A. (2012), "La evaluación entre iguales: beneficios y estrategias para su práctica en la universidad”, Revista de Educación, 359, 206-231.

JOHNSON, D. W., \& JOHNSON, R. T. (1987). "Learning together and alone: Cooperative, competitive, and individualistic learning“. Prentice-Hall, Inc.

LOPEZ, J. L. (2002). "Uso de rúbricas generalizadas para evaluar conocimientos en la técnica didáctica Aprendizaje Basado en Problemas." <www.mty.itesm.mx/rectoria/dda/rieee/word-files/1B- $\underline{\text { Uso- }}$

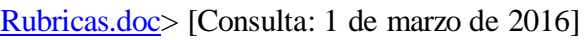

LOPEZ, V.M., MANRIQUE J.C. y VALLES C. (2011), "La evaluación y la calificación en los nuevos estudios de grado. Especial incidencia en la formación inicial del profesorado", Revista Electrónica Interuniversitaria de Formación del Profesorado, vol. 14, núm. 4, 57-72, <www.aufop.com/aufop/uploaded_files/articulos/1327436320.pdf> [Consulta: 25 de enero de 2016].

LOPEZ, V. M., MARTINEZ L.F. y JULIAN J.A. (2007). "La Red de Evaluación Formativa, Docencia Universitaria y Espacio Europeo de Educación Superior (EEES). Presentación del proyecto, grado de desarrollo y primeros resultados", Revista de Docencia Universitaria, vol. 1, núm. 2, 1-19,

<https://dialnet.unirioja.es/descarga/articulo/2739298.pdf > [Consulta: 25 de diciembre de 2015].

LOPEZ, V.M., FERNANDEZ J.M., SANTOS M.L. y FRAILE A. (2012), "Students' Self-Grading, Professor's Grading and Negotiated Final Grading at Three University Programmes: Analysis of reliability and grade difference ranges and tendencies", Assessment \& Evaluation in Higher Education, vol. 37, núm. 4, 453- 464.

MÉNDEZ, F. X., INGLES, C. J. e HIDALGO, M. D. (1999). "Propiedades psicométricas del Cuestionario de Confianza para Hablar en Público: Estudio con una muestra de alumnos de enseñanzas medias". Psicothema, 11, 65-74.

MINISTERIO DE EDUCACION, CULTURA Y DEPORTE (2003). "La integración del sistema universitario español en el Espacio Europeo de Enseñanza Superior. Documento marco." <http://www.uhu.es/convergencia_europea/documentos/documentos-2007/docmarco_MEC_feb2003.pdf> 
[Consulta: 2 de marzo de 2016]

MORALES, Z. y NAVARRO Y. (2013) "Evaluación de la competencia comunicativa "Hablar en Público" en un grupo de universitarios tras un curso sobre habilidades interpersonales." Revista de Enseñanza Universitaria, Julio 2013, 39

NÚÑEZ, H., CRESPO, E., ÚCAR, X., y BERÑE, A. L. (2014). "Enfoques De Evaluación Orientados A La Participación En Los Procesos De Acción Comunitaria.” Pedagogía social, (24), 79.

OLMEDO, E. M. (2013). "Enfoques de aprendizaje de los estudiantes y metodología docente: Evolución hacia el nuevo sistema de formación e interacción propuesta en el EEES. "Revista de Investigación Educativa, 31, 2, 411-429.

OREJUdO, S., NUÑO, J., FERNANDEZ, T., RAMOS, M. T. y HERRERO, M. L. (2006).

"Evolución del miedo a hablar en público en la Universidad. Variables personales y del entorno de enseñanza aprendizaje“. I Jornadas de Innovación docente, Tecnologías de la información y la comunicación e investigación educativa en la Universidad de Zaragoza.. BLOQUE TEMÁTICO 4. Investigación educativa. Noviembre 2006

<http://www.unizar.es/eees/innovacion06/COMUNIC_PUBLI/BLOQUE_IV/CAP_IV_22.pdf> [Consulta: 4 de enero de 2016]

PEREZ, A., JULIAN J.A. y LOPEZ V.M. (2009), "Evaluación formativa y compartida en el Espacio Europeo de Educación Superior (EEES)". En López, V. M. . Evaluación formativa y compartida en Educación Superior propuestas, técnicas, instrumentos y experiencias, España: Narcea. 19-43

RODRIGUEZ, G. IBARRA, M.S. y GARCIA, E. (2013) “Autoevaluación, evaluación entre iguales y coevaluación: conceptualización y práctica en las universidades españolas" Revista de Investigación en Educación, 11, 2. 〈http://reined.webs.uvigo.es/ojs/index.php/reined/article/view/708> [Consulta: 4 de enero de 2016]

SCARDAMALIA, M., y BEREITER, C. (1994). Development of Dialectical Processes in Composition. Language, Literacy, and Learning in Educational Practice: A Reader. USA: Multilingual Matters

STEIN, M. B., WALKER, J. R. y FORDE, D. R. (1996). "Public-speaking fears in a community sample: Prevalence, impact on functioning, and diagnostic classification". Archives of General Psychiatry, 53, 169-174. <http://www.ncbi.nlm.nih.gov/pubmed/8629892> [Consulta: 25 de diciembre de 2015].

UREÑA, N y RUIZ, E. (2012). "Experiencia de evaluación formativa y compartida en el Máster Universitario en Formación del Profesorado de Educación Secundaria" Psychology, Society \& Education, 4, 1, 29-44 <https://dialnet.unirioja.es/servlet/articulo?codigo=3961254> [Consulta: 2 de marzo de 2016].

WAMBA, A.M., RUIZ, C., CLIMENT, N. y FERRERAS, M. (2007). "Las rúbricas de evaluación de los Práctica como instrumento de reflexión para los estudiantes de Educación Primaria." A. Cid y col. (Coord.). En Actas del IX Simposium Internacional sobre prácticas. Prácticum y prácticas en empresas y en la formación universitaria. Buenas Prácticas en el Prácticum, 1251-1261. Poio (Pontevedra): AIDU (Asociación Iberoamericana de Didáctica Universitaria), U. de Vigo, y U. de Santiago de Compostela.

(cc) EY-NC-ND 2016, Universitat Politècnica de València 
Experiencia docente universitaria mediante el uso de metodologías activas para el aprendizaje como la evaluación formativa compartida y el trabajo de competencias transversales

RÚBRICA: Cada exposición será evaluada por el resto de grupos conforme a la siguiente plantilla: 50\% contenidos-50\% presentación

Plantilla de puntuación para la parte de Contenidos:

\begin{tabular}{|c|l|}
\hline Escala & \multicolumn{1}{c|}{ Descripción } \\
\hline 5 & $\begin{array}{l}\text { Se evidencia comprensión total del Trabajo. Incluye } \\
\text { todos los elementos requeridos en la actividad }\end{array}$ \\
\hline 3,75 & $\begin{array}{l}\text { Se evidencia comprensión del Trabajo. Incluye un alto } \\
\text { porcentaje de los elementos requeridos en la actividad }\end{array}$ \\
\hline 2,5 & $\begin{array}{l}\text { Se evidencia comprensión parcial del Trabajo. Incluye } \\
\text { algunos elementos requeridos en la actividad }\end{array}$ \\
\hline 1,25 & $\begin{array}{l}\text { Las evidencias indican poca comprensión del Trabajo. } \\
\text { No incluye los elementos requeridos en la actividad }\end{array}$ \\
\hline
\end{tabular}

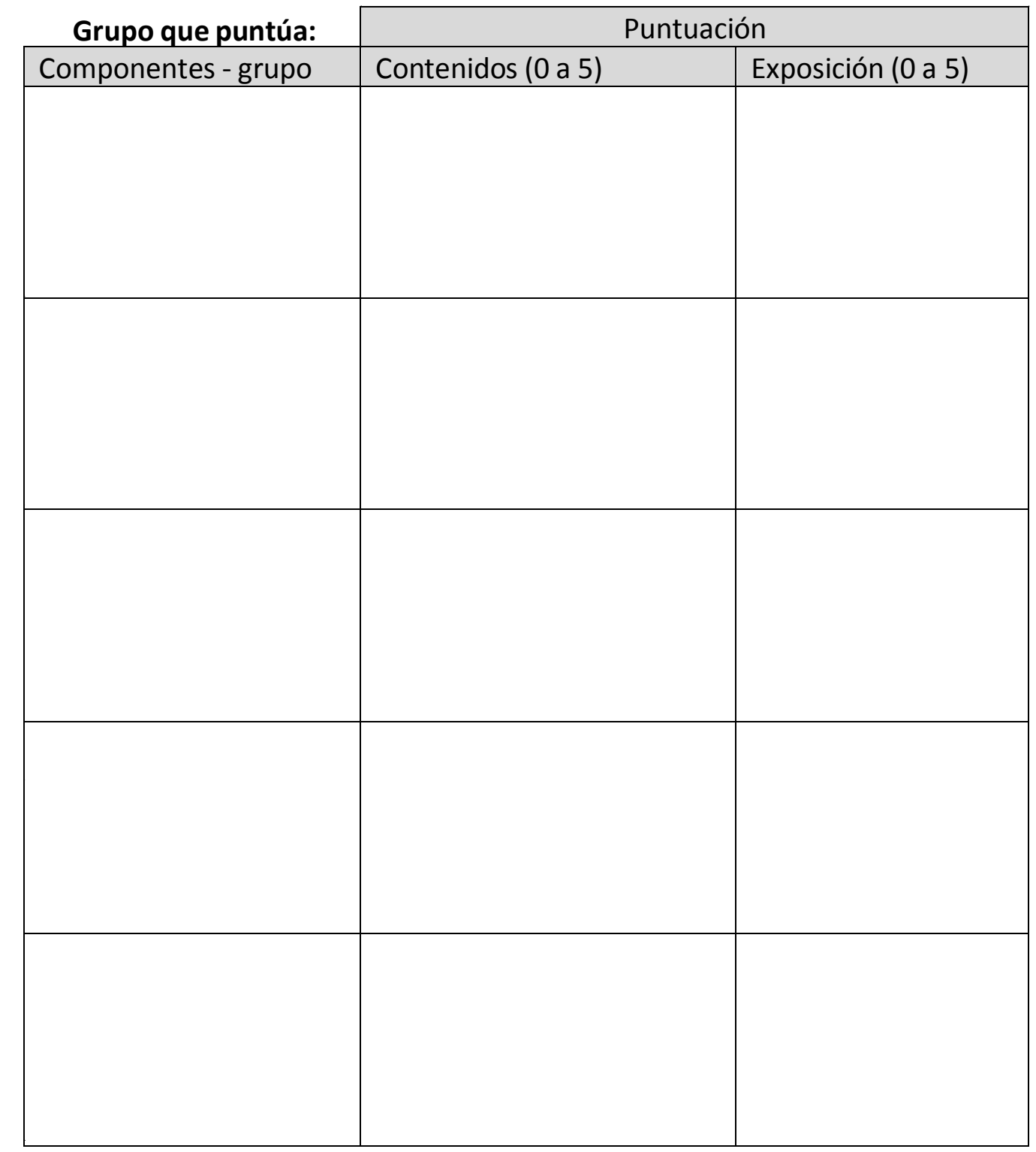

Plantilla de Puntuación para la parte de Exposición:

Escala $\quad$ Dominio de exposición y comunicación oral. Descripción La exposición cumple las normas ortográficas, expresa con claridad y orden las ideas, está correctamente presentada, resaltando la información pertinente, utiliza la información correctamente, se ciñe al tema aportando una perspectiva personal. El nivel de voz es el adecuado. El lenguaje corporal apoya toda la exposición

La exposición cumple las normas ortográficas, expresa con claridad y orden las ideas, está correctamente presentada

3,75 pero no aporta una perspectiva personal. El nivel de voz es adecuado durante casi toda la presentación. El lenguaje corporal apoya casi a toda la exposición.

La exposición incumple algunas normas ortográficas o no está expresada con claridad o no está correctamente presentada, no aporta una perspectiva personal. El nivel de voz es adecuado durante casi la mitad de la presentación. El lenguaje corporal apoya la mitad exposición.

No ha realizado la exposición o ésta presenta graves

problemas de ortografía, expresión, presentación, o bien no ha utilizado información pertinente o no se ciñe al tema propuesto. El nivel de voz es inadecuado durante casi toda la presentación. El lenguaje corporal casi no apoya exposición. 